\title{
ON A FIXED POINT THEOREM OF KIRK
}

\author{
CLAUDIO H. MORALES AND SIMBA A. MUTANGADURA
}

(Communicated by Palle E. T. Jorgensen)

\begin{abstract}
Let $X$ be a reflexive Banach space, $D$ an open and bounded subset of $X$, and $T: \bar{D} \rightarrow X$ a continuous mapping which is locally pseudocontractive on $D$. Suppose there exists an element $z \in D$ such that $\|z-T z\|<$ $\|x-T x\|$ for all $x$ on the boundary of $D$. Then under the so-called condition (S), $T$ has a fixed point in $D$. Although this result was proved earlier by Kirk, we show here a much easier approach.
\end{abstract}

Let $X$ be a (real) Banach space and let $D$ be a subset of $X$. An operator $T: D \rightarrow X$ is said to be $k$-pseudo-contractive $(k>0)$ (see [8]) if for each $x, y \in D$ and $\lambda>k$

$$
(\lambda-k)\|x-y\| \leq\|\lambda(x-y)-(T x-T y)\| .
$$

For $k=1(k<1)$ such mappings are said to be pseudo-contractive (respectively, strongly pseudo-contractive). One can easily derive the original definition of pseudo-contractive mappings, introduced by Browder [1], which states:

$$
\|x-y\| \leq\|(1+r)(x-y)-r(T x-T y)\|
$$

for all $x, y \in D$ and all $r>0$. Furthermore, the semi-inner product formulation (see Kato [5]) of this family of operators appears to be of significant use as well. This means that (2) may be described by

$$
\langle T x-T y, j\rangle \leq\|x-y\|^{2}
$$

for some $j \in J(x-y)$. The mapping $J: X \rightarrow 2^{X^{*}}$ is called the normalized duality mapping which is defined by

$$
J(x)=\left\{j \in X^{*}:\langle x, j\rangle=\|x\|^{2},\|j\|=\|x\|\right\} .
$$

Here $\langle\cdot, \cdot\rangle$ denotes the generalized duality pairing. It is easy to verify that nonexpansive mappings are pseudo-contractive. In addition, if the condition (1) holds locally, i.e., if each point $x \in D$ has a neighborhood $U$ such that the restriction of $T$ to $U$ is $k$-pseudo-contractive with (uniform) constant $k$, then $T$ is said to be locally $k$-pseudo-contractive.

The purpose of this paper is to provide an alternative proof of Theorem 1 of Kirk [6]. Kirk's proof is certainly ingenious, but it is also quite long

Received by the editors April 18, 1994.

1991 Mathematics Subject Classification. Primary 47H10.

Key words and phrases. Locally pseudo-contractive, convex hull, reflexivity. 
and involved. To the contrary, we offer a different approach which, to our knowledge, appears to be significantly much shorter. Other results are also simplified.

To fix our notation, we will denote the closure and boundary of $D$ by $\bar{D}$ and $\partial D$ respectively, and for $u, v \in X$ we use $\operatorname{seg}[u, v]$ to denote the segment $\{t u+(1-t) v: t \in[0,1]\}$. We will also use $B(x ; r)$ and $\bar{B}(x ; r)$ to stand for the open ball $\{z \in X:\|x-z\|<r\}$ and the closed ball $\{z \in X:\|x-z\| \leq r\}$ respectively. We denote the distance between the sets $A$ and $B$ by $\operatorname{dist}(A, B)$, and define it by

$$
\operatorname{dist}(A, B)=\inf \{\|a-b\|: a \in A, b \in B\} .
$$

The results of this paper are formulated either in arbitrary Banach spaces or, for stronger conclusions, in spaces in which the class of locally nonexpansive mappings enjoys some kind of smoothness. To be more precise, suppose $G$ is a bounded open subset of a normed space $X$ and $f$ is an arbitrary locally nonexpansive mapping from $G$ into $X$. We say that the space $X$ satisfies condition $(\mathbf{S})$ if for each $\varepsilon>0$ sufficiently small, there exists a largest $\xi(\varepsilon) \in$ $(0, \varepsilon]$ such that if $u, v \in G$ for which $\operatorname{seg}[u, v] \subset G$, then the conditions $\|u-f(u)\| \leq \xi(\varepsilon)$ and $\|v-f(v)\| \leq \xi(\varepsilon)$ imply $\|m-f(m)\| \leq \varepsilon$ for all $m \in \operatorname{seg}[u, v]$. We should note that $\xi$ also depends on $G$. Due to a result that implicitly appears in Browder [2] and Göhde [4], it can easily be shown that every uniformly convex space satisfies this condition $(\mathbf{S})$.

Now, we begin with a result for local contraction under an apparently new boundary condition.

Proposition 1. Let $X$ be a Banach space, let $K$ be a closed subset of $X$, and let $D$ be an open subset of $X$ with $D \cap K \neq \varnothing$. Suppose $T: \overline{D \cap K} \rightarrow K$ is a closed mapping which is a local contraction (with constant $k$ ) on $G=D \cap K$, and suppose there exists $z \in G$ such that

$$
\|z-T z\|<\|x-T x\|+(1-k)\|x-z\| \text { for } x \in \partial_{K} D .
$$

Then $\left\{T^{n}(z)\right.$ \} lies in $G$ and converges to a fixed point of $T$.

Proof. We first prove that $\operatorname{seg}[z, T z] \subset G$. To this end, we assume the contrary. Then there exists $m \in \operatorname{seg}[z, T z] \cap \partial_{K} D$ for which $\operatorname{seg}[z, m) \subset G$. Since $T$ is a global contraction on $\operatorname{seg}[z, m]$, we have

$$
\begin{aligned}
\|m-T m\| & \leq\|m-T z\|+\|T z-T m\| \\
& \leq\|z-T z\|-\|z-m\|+k\|z-m\| \\
& =\|z-T z\|-(1-k)\|z-m\| .
\end{aligned}
$$

This contradicts $(3)$ since $m \in \partial_{K} D$. Therefore, $\operatorname{seg}[z, T z] \subset G$. By replacing $z$ by $T z$, condition (3) also holds. This means $T^{n}(z) \in G$ for all $n \in \mathbb{N}$, while a standard argument shows that $\left\{T^{n}(z)\right\}$ is a Cauchy sequence.

Corollary 1. Let $X$ and $K$ be as in Proposition 1, and let $D$ be a bounded open subset of $X$. Let $T: \overline{D \cap K} \rightarrow K$ be a closed mapping which is locally nonexpansive on $G=D \cap K(\neq \varnothing)$, and suppose there exists $z \in G$ such that

$$
\|z-T z\|<\|x-T x\| \text { for all } x \in \partial_{K} D .
$$

Then $\inf \{\|x-T x\|: x \in G\}=0$. 
Proof. For each $t \in(0,1)$, the mapping $T_{t}$ defined by $T_{t}(x)=(1-t) z+t T(x)$ is a local contraction on $G$, and

$$
\left\|z-T_{t}(z)\right\|<\left\|x-T_{t}(x)\right\|+(1-t)\|x-z\|
$$

for $x \in \partial_{K} D$. Then $\left\{T_{t}^{n}(z)\right\}$ lies in $G$, and due to the proof of Proposition 1, $\inf \left\{\left\|x-T_{t}(x)\right\|: x \in G\right\}=0$ for each $t \in(0,1)$. Since $D$ is bounded and also

$$
x-T_{t}(x)=(1-t)(x-z)+t(x-T x)
$$

for $x \in G$ and $t \in(0,1)$, it follows that $\inf \{\|x-T x\|: x \in G\}=0$.

Before we state the main theorem of this paper, we prove some preliminary facts. For the sake of clarity, we will use $G_{\beta}$ and $C\left(G_{\beta}\right)$ to denote the set $\{x \in D \cap K:\|x-T x\|<\beta\}$ and a connected component of $G_{\beta}$ respectively.

Lemma. Let $X$ be a Banach space which satisfies the condition (S), let $K$ be a closed convex subset of $X$, and let $D$ be a bounded open subset of $X$ with $D \cap K \neq \varnothing$. Suppose $T: D \cap K \rightarrow K$ is a local nonexpansive mapping. Furthermore, assume that the set $G_{\beta_{0}}$ is nonempty for some positive number $\beta_{0}$ and $\operatorname{dist}\left(\partial_{K} G_{\beta_{0}}, \partial_{K} D\right)>0$. Then there exists a positive number $\beta_{1}<\beta_{0}$ for which

$$
\operatorname{seg}[u, v] \subset G_{\beta_{0}} \text { for } u, v \in C\left(G_{\beta_{1}}\right) .
$$

Proof. Since $G_{\beta_{0}}$ is open in $K$, there exists an open set $D_{0}$ in $D$ so that $G_{\beta_{0}}=D_{0} \cap K$. Also, since $G_{\beta_{0}}$ is nonempty, there is an element $z \in G_{\beta_{0}}$ for which condition (4) holds on $\partial_{K} D_{0}$. This means that we may select $0<\alpha_{0}<\beta_{0}$ and $\beta_{1}=\xi\left(\alpha_{0}\right)$, where the set $G_{\beta_{1}}=\left\{x \in G_{\beta_{0}}:\|x-T x\|<\beta_{1}\right\}$ is clearly nonempty due to Corollary 1 . We shall show now that (5) holds. To see this, let $u \in G_{\beta_{1}}$ and define the set

$$
G_{\beta_{1}}(u)=\left\{v \in G_{\beta_{1}}: \operatorname{seg}[u, v] \subset G_{\beta_{0}}\right\} .
$$

We may assume, without loss of generality, that $G_{\beta_{1}}$ is a connected component of $G_{\beta_{0}}$. Then we claim that $G_{\beta_{1}}(u)=G_{\beta_{1}}$. Otherwise, we may assume there exists $w \in G_{\beta_{1}} \backslash G_{\beta_{1}}(u)$. Since $G_{\beta_{1}}$ is path-connected, there is a path $\gamma$ in $G_{\beta_{1}}$ that joins $u$ with $w$. This means that there exists $v \in \partial_{K} G_{\beta_{1}}(u) \cap G_{1}$ so that $\operatorname{seg}[u, v] \in \overline{G_{\beta_{0}}}$. We shall show now that $\operatorname{seg}[u, v] \subset G_{\beta_{0}}$. To see this, let $x \in \operatorname{seg}[u, v]$. Then, since $u$ and $v$ are in $G_{\beta_{1}}$, the condition (S) implies that $\|x-T x\| \leq \alpha_{0}<\beta_{0}$, which means $x \in G_{\beta_{0}}$. Therefore $v \in G_{\beta_{1}}(u)$, and thus $G_{\beta_{1}}(u)=G_{\beta_{1}}$. This completes the proof.

Next, we give a much simpler proof of Theorem 1 of [7], where just a particular case of it will be used in our main theorem.

Proposition 2. Let $X$ be a reflexive Banach space, and let $K$ and $D$ be as in the previous lemma. Let $T: \overline{D \cap K} \rightarrow K$ be a closed mapping which is locally nonexpansive on $G=D \cap K$, and suppose the following conditions hold:

(i) There exists $z \in G$ such that $\|z-T z\|<\|x-T x\|$ for all $x \in \partial_{K} D$.

(ii) $X$ satisfies the condition (S) with respect to $T$.

Then $T$ has a fixed point in $G$.

Proof. We first observe, by Corollary 1 , that $\inf \{\|x-T x\|: x \in G\}=0$. Now, let $\beta_{0}=\|z-T z\|>0$, and let $0<\alpha_{0}<\beta_{0}$. We define a sequence $\left\{\beta_{n}\right\}$ by $\beta_{n}=\xi\left(\alpha_{n-1}\right)$, where the sequence $\left\{\alpha_{n}\right\}$ is chosen to be positive and satisfies 
the following properties: $\alpha_{n}<\beta_{n}$ for $n \in \mathbb{N}$ and $\alpha_{n} \rightarrow 0^{+}$. We now define the set

$$
G_{n}=\left\{x \in G:\|x-T x\|<\beta_{n}\right\}
$$

for $n=0,1,2, \ldots$. Then each $G_{n}$ is nonempty and open relative to $K$. Now, without loss of generality, we may assume that these sets are connected, and for each $n$, we select $x_{n} \in G_{n}$. In what follows, we let

$$
E_{n}=\operatorname{co}\left\{x_{k}: k \geq n+1\right\}
$$

where $\operatorname{co}(A)$ denotes the convex hull of $A$. Following [3], we claim that for $n \leq m$, the set

$$
E_{n, m}=\operatorname{co}\left\{x_{k}: n+1 \leq k \leq m+1\right\}
$$

is contained in $G_{n}$ for all $n \geq 0$. To this end, we proceed by induction. For $n=m$, the claim clearly holds. We suppose now that $E_{j, m} \subset G_{j}$ and let $x \in E_{j-1, m}$. Then we have

$$
x=\lambda x_{j}+(1-\lambda) y
$$

for some $y \in E_{j, m} \subset G_{j}$ and $\lambda \in[0,1]$. Therefore the Lemma and its proof imply that $x \in G_{j-1}$. Hence $E_{n, m} \subset G_{n}$ for all $m \geq n \geq 0$, and thus it follows that $E_{n} \subset G_{n}$. Since $\bar{E}_{n+1} \subset \bar{E}_{n}$ and $\bar{E}_{n}$ is weakly compact, we conclude

$$
\varnothing \neq \bigcap_{n=1}^{\infty} \bar{E}_{n} \subset \bigcap_{n=1}^{\infty} \bar{G}_{n} .
$$

This means that there is $x_{0} \in \bigcap_{n=1}^{\infty} \bar{G}_{n}$, which is a fixed point of $T$.

We now prove the main result of this paper.

Theorem 1. Let $X$ be a reflexive Banach space, which satisfies the condition $(\mathrm{S})$, and let $D$ be a bounded open subset of $X$. Suppose $T: \bar{D} \rightarrow X$ is a continuous mapping which is locally pseudo-contractive on $D$, and suppose there exists $z \in D$ such that

$$
\|z-T z\|<\|x-T x\| \text { for all } x \in \partial D .
$$

Then $T$ has a fixed point in $D$.

Proof. We may assume without loss of generality that $D$ is connected. Also, due to Proposition 2 of [10], the mapping $F x=2 x-T x$ is globally one-to-one on $D$. Therefore, in view of Theorem 2 of [9], we select $w \in D$ so that

$$
\|w-T w\|<\|z-T z\| .
$$

This means that if we define a subset $W$ of $D$ by

$$
W=\{x \in D:\|x-T x\|<\|z-T z\|\},
$$

then $\partial W \subset D$ and

$$
\|w-T w\|<\|x-T x\| \text { for } x \in \partial W .
$$

It follows that $\operatorname{seg}[w, F w] \subset F(W)$ (see Assertion 1 of [6]), which implies that $F^{-1}$ is nonexpansive on $\operatorname{seg}[w, F w]$, and thus

$$
\left\|w-F^{-1}(w)\right\|<\|x-T x\| \quad \text { for } x \in \partial W .
$$


Since $\partial F(W)=F(\partial W)$, we may say that for each $y \in \partial F(W)$, there exists $x \in \partial W$ such that $y=F(x)$ and

$$
\left\|w-F^{-1}(w)\right\|<\left\|y-F^{-1}(y)\right\| \text {. }
$$

Consequently, by Proposition $2, F^{-1}$ has a fixed point in $D$. This completes the proof.

Now, we may derive Theorem 1 of Kirk [6] as a consequence of Theorem 1.

Corollary 2. Let $X$ be uniformly convex, $D$ a bounded open subset of $X$, and $T: \bar{D} \rightarrow X$ a continuous mapping which is locally pseudo-contractive on $D$. Suppose there exists $z \in D$ such that

$$
\|z-T z\|<\|x-T x\| \text { for all } x \in \partial D .
$$

Then $T$ has a fixed point in $D$.

\section{REFERENCES}

1. F. E. Browder, Nonlinear mappings of nonexpansive and accretive type in Banach spaces, Buli. Amer. Math. Soc. 73 (1967), 875-882.

2. Semicontractive and semiaccretive nonlinear mapping in Banach spaces, Bull. Amer. Math. Soc. 74 (1968), 660-665.

3. T. H. Chang and C. L. Yen, Some fixed point theorems in Banach spaces, J. Math. Anal. Appl. 138 (1989), 550-558.

4. D. Göhde, Zum frinzip der Kontraktiven Abbildung, Math. Nachr. 30 (1965), 251-258.

5. T. Kato, Nonlinear semigroups and evolution equations, J. Math. Soc. Japan 19 (1967), 508-520.

6. W. A. Kirk, A fuxed pint theorem for local pseudo-contractions in uniformly convex spaces, Manuscripta Math. 30 (1979), 89-102.

7. __ Locally nonexpansive mappings in Banach spaces, Fixed Point Theory (E. Fadell and G. Fournier, eds.), Lecture Notes in Math., vol. 886, Springer-Verlag, Berlin and New York, 1981, pp. 178-198.

8. C. H. Morales, Pseudo-contractive mappings and the Leray-Schauder boundary condition, Comment. Math. Univ. Carolin. 20 (1979), 745-756.

9. $\ldots$, On the fuxed-point theory for local k-pseudocontractions, Proc. Amer. Math. Soc. 81 (1981), 71-74.

10. C. H. Morales and S. A. Mutangadura, On the approximation of fixed points for locally pseudo-contractive mappings, Proc. Amer. Math. Soc. 123 (1995), 417-423.

Department of Mathematics, University of Alabama in Huntsville, Huntsville, Alabama 35899

E-mail address: morales@math.uah.edu

Department of Physics, University of Zimbabwe, HaRare, Zimbabwe

E-mail address: mutanga@zimbix.uz.zw 